\title{
Therapeutic plasma exchange (TPE) for semi-critical neurology presentations in a non-acute neurology set-up: clinical practice and challenges
}

\author{
Keng Seng Fu, Pei Yin Wong, Fu Liong Hiew (D)
}

To cite: Fu KS, Wong PY, Hiew FL. Therapeutic plasma exchange (TPE) for semi-critical neurology presentations in a non-acute neurology set-up: clinical practice and challenges. BMJ Neurology Open 2020;2:e000020. doi:10.1136/ bmjno-2019-000020

Received 18 November 2019 Revised 02 January 2020 Accepted 19 January 2020
Check for updates

(C) Author(s) (or their employer(s)) 2020. Re-use permitted under CC BY-NC. No commercial re-use. See rights and permissions. Published by BMJ.

Department of Neurology, Hospital Kuala Lumpur, Kuala Lumpur, Wilayah Persekutuan, Malaysia

Correspondence to

Dr Fu Liong Hiew;

hiewf|@gmail.com

\section{ABSTRACT}

Introduction Therapeutic plasma exchange (TPE) for semi-critical neurological manifestations can be managed in non-acute setting instead of critical care unit. In 2014, we established a non-acute neurology TPE unit for semicritical haemodynamically stable patients. In this study, we aimed to evaluate the technical and safety parameters from the first 3 years of service.

Materials and methods We analysed prospectively collected TPE data for patients treated with centrifugation TPE at our non-acute neurology TPE unit in Kuala Lumpur Hospital between May 2015 and June 2018.

Results A total of 245 TPE procedures were performed in 55 patients for nine neurological indications, predominantly the central nervous system (79\%). Twenty four per cent $(n=13)$ had category I and $73 \%(n=40)$ had category II indication (American Society for Apheresis (ASFA) 2019). Others (4\%) were not in ASFA indications. Neuromyelitis optica spectrum disorders accounted for half $(51 \%)$ of the total patients. Twenty-three (41.8\%) patients experienced adverse events, with hypotensive episodes being the the most common ( $n=12 / 55,21.8 \%$ ). Five $(9.1 \%)$ patients had catheter-related blood stream infection, correlating with higher exchange plasma volume $(p=0.023)$. Symptomatic hypocalcaemia was less common $(n=5 / 55,9.1 \%)$ and allergic reaction to human albumin was rare $(n=1 / 55,1.8 \%)$. Four technical errors detected. Three involved centrifugation sets manufacturing defects and one involved error in centrifugation set installation. Seven $(2.9 \%)$ procedures were terminated: 5 for adverse effects and 2 for technical errors.

Conclusion Performing TPE among semi-critical patients with neurology manifestations in basic non-acute set-up proved safe, with predictable complications. This set-up reduced the reliance on critical care services for TPE procedures.

\section{INTRODUCTION}

Therapeutic plasma exchange (TPE) is a technique using extracorporeal circuit to separate and remove circulating large molecular weight protein-bound substances such as pathological antibodies and immune complexes, replacing it with fluids such as albumin, fresh frozen plasma (FFP), colloids or crystalloids. ${ }^{1}$ TPE can be performed using centrifugation system or membrane plasma separation devices via a temporary peripheral or central venous catheter vascular access. ${ }^{23}$ Traditionally, TPE was used mainly in the treatment of patients with haematological conditions such as Waldenstrom's macroglobulinaemia. With expanding indications, TPE is now used in many neurological diseases as well such as neuromyelitis optica spectrum disorders (NMOSD), myasthenia gravis (MG) and Guillain-Barre syndrome (GBS). ${ }^{4}$ Majority of the patients with these neurological disorders present acutely, with a significant proportion of them in critical condition, requiring intensive care unit (ICU) admission due to haemodynamic instability or respiratory failure..$^{5-7}$ Therefore, TPE procedures are frequently performed in the ICU setting for conditions with increased risks associated with the procedure. ${ }^{8}$

However, critical care set-up varies between centres, and in most institutions including ours, availability of critical care beds was and continues to be limited. This practice system required TPE to be performed in critical care units with support from other subspecialty disciplines such as nephrology and haematology; thus it was time and resources consuming. More importantly, semi-critical neurology patients with stable haemodynamics may receive TPE treatment in a nonacute setting with proper monitoring by trained staffs. In addition, neurology set-up in our institution lacked critical care beds, and there was no mobile TPE support available outside critical care set-up. Thus, being cognizant of these limitations, our department established an in-house non-acute TPE unit using single centrifugation device in 2014, supported by a local TPE team comprised of trained neurology nurses and neurologists. ${ }^{9}$ This TPE unit was designed to support TPE procedures with basic vital 
signs monitoring equipment on haemodynamically stable neurology patients without respiratory failure. A group of trained neurology nurses from our department was the key service providers who performed all TPE procedures.

As TPE involves bulk removal of plasma volume (PV), patients with neurological conditions experience potential risks of procedure-related complications such as haemodynamic instability, electrolyte imbalances and catheter-related blood stream infections (CRBSI) ${ }^{10}$ In addition, data on technical aspects of performing TPE are essential to ensure the delivery of successful, safe and efficient TPE. Therefore, the local TPE team initiated a TPE database soon after the commencement of the service, registering data on indications, technical aspects and safety profiles as part of the strategies for future improvement. Such data will also enable us to compare our TPE management with other centres. In this study, we aimed to report the data from the first 3 years of the database, and to evaluate several crucial service parameters, comparing with recommended standards from other centres and international guidelines.

\section{MATERIALS AND METHODS Design and setting}

We included all consecutive adult neurology patients who received TPE in our non-acute neurology TPE unit, Kuala Lumpur Hospital between May 2015 and June 2018. This was a non-interventional analysis of prospectively collected data on TPE patients under our local demyelnating TPE database.

The neurology department, Kuala Lumpur Hospital is the largest tertiary neurology referral centre in the country under the provision of Ministry of Health, Malaysia. Our general neurology ward was equipped with 40 beds, including four in the acute bay that admits close to 2000 neurology patients per year. We converted a non-acute single bedded air-conditioned room into a TPE suit, equipped with a centrifugal TPE machine and vital sign monitoring equipment. Full TPE unit set-up and protocol are available on our previous publication elsewhere. ${ }^{9}$ Patients requiring TPE therapy were assessed and stratified by treating designated TPE neurologists and the TPE team. TPE prescription was decided by the treating neurologist in accordance with our local TPE guidelines. Our TPE unit performed TPE on patients with semicritical neurological presentations that did not require ventilator or ICU support. Criteria for eligible patients are as stated in table 1.

\section{TPE technique}

TPE procedures were performed by a dedicated TPE team, which included experienced staff nurses, specialist registrars and consultant neurologists. We used Spectra Optia centrifugation system by Terumo BCT. All patients had corrected basic blood parameters (complete blood counts, renal profile, liver profiles, coagulation profile and serum calcium) prior to commencement of every
Table 1 Criteria of semi-critical acute neurology patients for TPE in non-acute setting

\begin{tabular}{ll}
\hline No & Criteria \\
\hline 1 & Haemodynamically stable without inotropic support \\
2 & No respiratory failure or requiring ventilatory support \\
3 & $\begin{array}{l}\text { No clinical or laboratory evidence of systemic } \\
\text { infection }\end{array}$ \\
4 & $\begin{array}{l}\text { No coagulation disorders or electrolyte imbalance } \\
\text { No organ failure noted on admission such as acute } \\
\text { kidney injury requiring renal replacement therapy }\end{array}$ \\
\hline
\end{tabular}

TPE, therapeutic plasma exchange.

TPE procedures. In our centre, all vascular accesses were by a temporary double lumen central venous catheter $(11.5 \mathrm{Fr} / 16 \mathrm{~cm}, 12 \mathrm{Fr} / 15 \mathrm{~cm})$ inserted by interventional radiologists under ultrasound guidance to minimise procedure-related complications. In a typical treatment course, 4-6 sessions of exchanges were performed 24 hours apart. Plasma exchange volume was calculated based on individual patient's weight and haematocrit (Hct) value. PVs were calculated using the formula ${ }^{11}$ $\mathrm{PV}=(1-\mathrm{Hct})(\mathrm{b}+\mathrm{cW})$ : wherein the Hct is expressed as a fraction (ie, range $0-1$ ); $b$ is a constant of 1530 for males, 864 for females; c is a constant of 41 for males, 47.9 for females; and $\mathrm{W}$ is the dry weight expressed in kilograms. Patients were pre-loaded with $500 \mathrm{~mL}$ of normal saline to ensure adequate hydration and volume before each procedure. The blood pump speed was set between 40 and $120 \mathrm{~mL} / \mathrm{min}$. Anticoagulation was performed with continuous infusion of sterile acid-citrate-dextrose solution United States Pharmacopeia (USP) formula A in accordance to the manufacturer recommendation of infusion rate during the TPE procedure. Intravenous $1 \mathrm{~g}$ calcium gluconate was infused during TPE as standard protocol. Replacement fluids were $2 / 3$ albumin $5 \%$ in Hartmann's solution and remaining $1 / 3$ with normal saline crystalloids solution. Vital signs monitoring every 15 min during the procedures with a cardiac monitoring were performed as standard observation protocol (15-30 min intervals). Informed consent was obtained from all patients before commencing on TPE procedure with explanation on the associated procedure-related risks. All patients prior to procedure had pre-procedure counselling and were given in-house developed brochures on TPE in two languages, that is, English and Malay.

\section{Data collection and analysis}

We analysed baseline patient characteristics including demographic, neurological disorders, indication for TPE and all data related to TPE procedures including Hct levels, total TPE treatment time (set-up + preparation + priming times and actual procedure time), technical parameters and adverse events. Cases were classified according to indication categories I-IV by American Society for Apheresis (ASFA). Adverse events were graded according to the 
Table 2 Grading of adverse events in therapeutic plasma exchange $^{12}$

\begin{tabular}{|c|c|c|}
\hline Grade & Quality & Intervention \\
\hline I & Mild & No intervention required \\
\hline II & Moderate & $\begin{array}{l}\text { Intervention required; } \\
\text { treatment completed }\end{array}$ \\
\hline III & Severe & $\begin{array}{l}\text { Procedure interrupted or } \\
\text { abandoned }\end{array}$ \\
\hline IV & Fatal & Patient expired \\
\hline
\end{tabular}

grading adapted from the Swedish Apheresis Group ${ }^{12}$ (table 2). Termination rate of TPE due to adverse events, technical or human error was determined.

\section{Statistical analysis}

Simple descriptive statistics were used to analyse the results. Data analyses were performed using SPSS V.22. Results are described as medians and IQR (25th-75th percentiles) for quantitative variables or numbers and percentages for qualitative variables. Comparison of means was performed using Student's t-test. Significance level was set at $p$ values $<0.05$. Pearson correlation tests were performed to identify the association of complications related to TPE with demographics and technical TPE parameters. Due to the uneven distribution of neurological diseases and our small sample size, some results were not tested for significance.

\section{RESULTS}

A total of 55 Malaysian patients of mixed race (25 Malays, 45\%; 28 Chinese, 51\%; 2 Indian, 4\%) underwent 245 centrifugation technique TPE procedures during this study period of 3 years. There were 16 (29\%) men and $39(71 \%)$ women with median age of 41 years old (range 13-63). There was no significant different between age by gender $(\mathrm{p}=0.116)$. Table 3 shows the descriptive statistics for age, gender and primary diagnosis of indication for TPE treatment summarised as the number of patients (percentage), median (range) and mean (SD).

Clinical indications for TPE are as illustrated in figure 1. Twenty four per cent $(13 / 55)$ of the patients belonged to category I and $73 \%(40 / 55)$ were in category II indication for TPE according to the ASFA 2019 guidelines. Four per cent $(2 / 55)$ had chronic relapsing inflammatory optic neuropathy (CRION) and was not categorised by ASFA as of 2019. We identified nine neurological indications for TPE from both central and peripheral nervous system (PNS) disorders. NMOSD accounted for half (51\%) of the total number of patients received TPE therapy, majority of them $(82 \%)$ being women. This was followed by patients with idiopathic inflammatory transverse myelitis (IITM) (eight patients, 15\%), similarly affecting greater proportion of female patients $(75 \%)$. All patients with NMOSD and IITM received corticosteroids therapy, however, with partial response. Other central nervous system (CNS) disorders included multiple sclerosis (MS) (3, 5\%), CRION (2, 4\%), acute disseminated encephalomyelitis (ADEM) $(1,2 \%)$ and $\mathrm{N}$-methyl-D-aspatartate receptors encephalitis refractory to corticosteroid therapy $(1,2 \%)$. For PNS and neuromuscular junction (NMJ) disorders, GBS $(6,11 \%)$ and MG $(4,7 \%)$ were the main indications for TPE. All patients with GBS and MG had partially responded to intravenous immunoglobulin (Ig) given as initial therapy. Two patients with chronic inflammatory demyelinating polyneuropathy (CIDP) had TPE due to acute worsening of weakness refractory to corticosteroids and intravenous Ig therapy.

The mean number of TPE procedures performed per patient was 4.5 cycles (SD 1.2). Eighty seven per cent $(48 / 55)$ of the patients had at least 4 cycles of TPE procedures performed. This comprised of 40 patients who had 5 cycles of TPE, 1 patient with extended 6 cycles of TPE and 7 patients who received 4 cycles of TPE. Remaining $7(13 \%)$ patients had less than 4 cycles of TPE. Overall procedure termination rate was $2.9 \%$ ( 7 procedures, 5 for adverse effects and 2 for technical errors). The median weight of patients was $56 \mathrm{~kg}(36-111 \mathrm{~kg})$ and the median Hct was $36 \%$ (28\%-48\%). The median PV exchange per procedure was $2245 \mathrm{~mL}$ (range $1720-3455 \mathrm{~mL}$ ), corresponding to $1.0(0.9-1.5)$ times the estimated plasma volume (EPV) based according to patient's body weight and Hct. The mean total treatment time was $87.1 \mathrm{~min}$ (SD 9.0), with mean actual treatment time recorded at $56.9 \mathrm{~min}$ (SD 9.7). Close to 8\% (19/245) of the procedures performed had PVs exchanged below 1.0 times of patient's EPV. Median Hct of patients who had PV exchanged below 1.0 times EPV was significantly lower than those with higher plasma exchange volume $(32 \%$ vs $36 \%, \mathrm{p}<0.005)$. However, there was no difference in terms of median weight among the two groups of patients (52 kg vs $56 \mathrm{~kg}, \mathrm{p}=0.388$ ).

All patients had internal jugular catheter inserted for TPE under ultrasound guidance. No immediate complications were recorded following the procedures in all patients. Twenty three of the 245 plasma exchange procedures were reported to have at least one adverse event, equivalent to a complication rate of $9.4 \%$. Adverse events were graded as mild (grade I) in $5.3 \%$ (13/245), moderate (grade II) in $2.0 \%(5 / 245)$ and severe (grade III) in $2.0 \%$ (5/245). Figure 2 shows the distribution of adverse events over 3 years in relation to number of TPE procedures. Hypotensive episodes during TPE were the most commonly reported events, occurring in 12 (4.9\%) of the patients. All patients responded to either with reduction of inlet flow rates, intravenous hydration or cessation of TPE procedure. One patient needed commencement of intravenous infusion of vasopressors. Half (6/12) of the number of hypotensive episodes developed during TPE was reported during the first years of TPE service commencement, corresponding to incidence rate of $9.2 \%$. As of the last 1 year, only two patients were reported to develop hypotensive events $(2.6 \%)$. Six $(2.4 \%)$ patients had CRBSI following insertion of central venous catheter. 


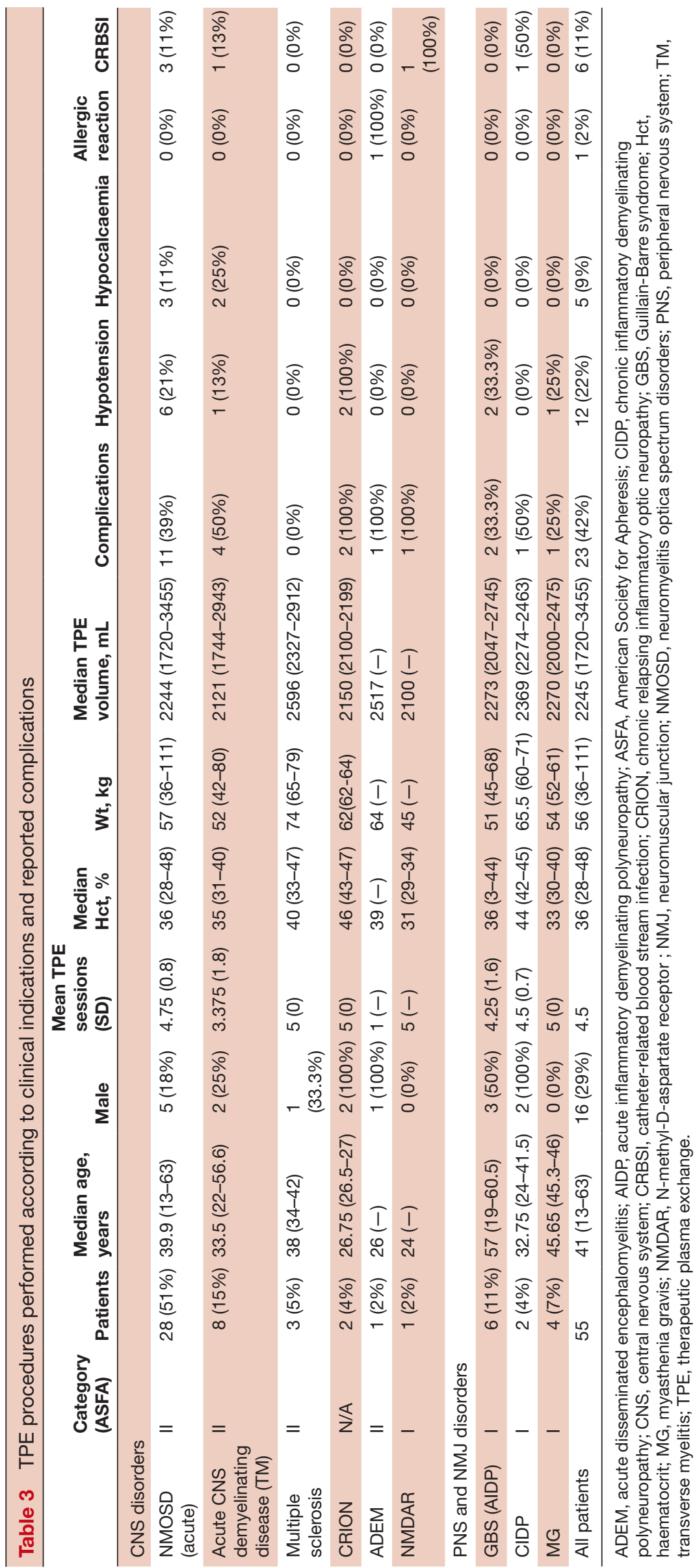




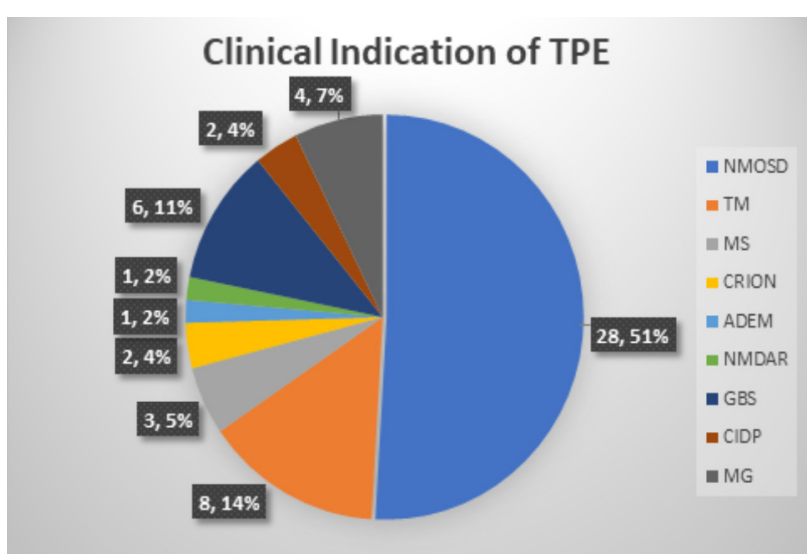

Figure 1 Clinical indications of therapeutic plasma exchange (TPE). ADEM, acute disseminated encephalomyelitis; CIDP, chronic inflammatory demyelinating polyneuropathy; CRION, chronic relapsing inflammatory optic neuropathy; GBS, Guillain-Barre syndrome; MG, myasthenia gravis; MS, multiple sclerosis; NMOSD, neuromyelitis optica spectrum disorders; TM, transverse myelitis.

All of them were successfully treated with systemic intravenous antibiotics. Symptomatic hypocalcaemia associated with the acid-citrate-dextrose solution use was observed in $5(2 \%)$ of the patients, while allergic reactions associated with the use of transfused human albumin such as rashes and nausea were reported in $1(0.4 \%)$ patient. There was no potentially life-threatening complications or procedure-related mortality occurring among our cohort of patients.

Four incidences involving technical errors were noted by TPE staffs while performing the procedures; three were due to centrifugation set manufacturing defects, resulting in inability to initiate procedures (two sets) and procedure termination due to high pressure error (one set). Another technical glitch was due to error in centrifugation set installation onto the machine. The high centrifugation frequency resulted in a crack at the tubing set, with subsequent blood leak and visible contamination in the rotating separation chamber. No report of adverse event affecting the patient was noted.

We performed further Pearson's correlation analysis to search for associations of TPE complications with procedure parameters (baseline weight, pre-procedure Hct, number of TPE cycles and exchanged PV). We found only higher PV exchange was significantly associated with CRBSI $(p=0.023)$. Although the exchanged PV was based

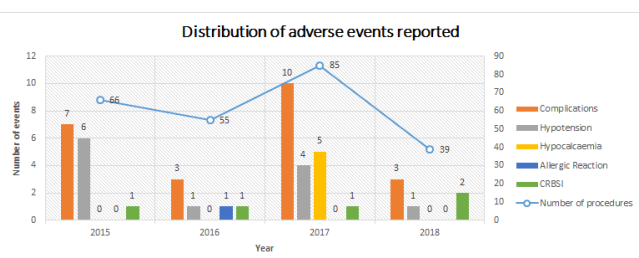

Figure 2 Distribution of adverse events in relation to number of therapeutic plasma exchange procedures. CRBSI, catheter-related blood stream infection. on formula calculated with patient's body weight and preprocedure Hct, both parameters were not independently a factor for higher infection risk. Similarly, we found no different in average Hct among the group of patients who developed hypotension and those who did not $(37 \%$ vs $37 \%, \mathrm{p}=0.889$ ). Number of TPE cycles was not associated with the incidence of infection. No TPE parameter was found to be associated with hypotension, hypocalcaemia and allergic reaction.

\section{DISCUSSION}

Advances in TPE technology since its first development in the 1960s have resulted in breakthroughs in treatment of increasing numbers of disorders, including in several immune-mediated neurological diseases. In our local neurology setting, TPE has recently become a more commonly used immunotherapy primarily due to the expanding clinical indications, better accessibility and significantly lower treatment cost compared with other treatment options such as intravenous Ig or monoclonal antibodies.

At initial set-up of our non-acute TPE unit, we performed TPE for various central and PNS disorders, similar to other recently published data from established centres. ${ }^{13-15}$ With time, we observed an increase in TPE applications for CNS disorders indications. Of all TPE procedures performed during these study periods, $73 \%$ were category II CNS indications and $2 \%$ category I CNS indications based on ASFA guidelines. Remaining 20\% were category I PNS indications. Recent advances in the understanding of immunopathogenicity of various CNS disorders such as NMOSD, MS, ADEM and NMDAR encephalitis has contributed significantly to the change in the panorama of TPE use. We performed lesser number of TPE for patients with PNS in our non-acute neurology TPE unit compared with other major TPE centres. This was due to two main factors: local treatment protocols and patient selection. A large majority $(75 \%)$ of patients with GBS in our centre were treated with intravenous Ig during acute presentation. ${ }^{16}$ TPE was reserved for those refractory cases requiring prolonged mechanical ventilation, often in the ICU setting. These patients were not included in this analysis. Physician's choice of favouring intravenous Ig for treatment in GBS was also observed in more developed countries. ${ }^{12}$ The same treatment protocol was applied to MG patients admitted to our centre with myasthenic crisis. For patients with CIDP, treatments were primarily with corticosteroids and intravenous Ig. ${ }^{17}$ The use of TPE was limited due to the chronicity of the disease. Notably, two $(4 \%)$ of our patients with CRION received TPE therapy following acute relapse of severe optic neuritis with poor response to corticosteroids therapy. However, CRION has not been listed as an indication under ASFA. The rationale for TPE was based on the postulation that CRION is immune-mediated optic neuritis similar to NMOSD, despite no particular pathogenic antibody thus far found in these patients. ${ }^{18}$ 
The observed overall complication rates at our TPE unit $(9.4 \%)$ were comparable with the recently reported experience in most centres and of the World Apheresis Association (WAA) apheresis registry, ranging from 5\% to $12 \% .{ }^{10131419}$ We observed no complications related to procedures for central venous vascular access, performed under ultrasound-guided by in-house interventional radiologists. In our study, TPE procedures performed by neurology nursing staffs were safe, well-tolerated and adverse events were for the most part mild to moderate. The spectrum of adverse events observed was similar to most reported series. ${ }^{10}$ However, we experienced slightly higher rates of hypotension $(4.9 \%)$ secondary to fluid shift compared with the reported rates at $0.4 \%-4.2 \%{ }^{10}$ This could be attributed to the use of a less vascular expansion fluid replacement regime of human albumin $70 \%$ and crystalloids $30 \%$ compared with $100 \%$ human albumin. ${ }^{3}$ Majority of the hypotensive events were handled by reducing inlet flow rates and infusion of crystalloid saline. The number of patients who developed CRBSI was low with no life-threatening events seen. All bacteraemia responded to intravenous antibiotic therapies. With pre-procedure, serum calcium check and infusion of $1 \mathrm{~g}$ calcium gluconate during TPE as standard treatment protocol, citrate anti-coagulant-derived symptomatic hypocalcaemia (paresthesia, nausea and emesis) were uncommon at our centre. In addition, the risk was further reduced by having human albumin and crystalloids as fluid replacement instead of FFP. ${ }^{320}$ Minor and unpredictable complications such as allergic reactions to human albumin were rare. Despite reported reduction of clotting factors associated with the use of human albumin as plasma replacement and some platelets loss with the centrifugation system which increased the risk of haemorrhage, this has not been demonstrated in our study. ${ }^{3}$

The median PV exchange per procedure at $2245 \mathrm{~mL}$ among our patients was lower compared with other centres performing TPE for neurological indications. ${ }^{21-23}$ The corresponding relative PV for our TPE procedures was $1.0(0.9-1.5)$ times the EPV. The lower median plasma exchange volume was the main factor for shorter mean treatment time of $56.9 \mathrm{~min}$, compared with similar study using centrifugal TPE from Croatia (mean $91 \mathrm{~min}$ ) and Germany $(120 \mathrm{~min}){ }^{22}{ }^{23}$ The relative PV from our analysis was lower compared with the plasma exchange volume for neurology indications in Europe, documented at $1.17(0.97-1.38) .{ }^{21}$ This was likely due to lower baseline Hct (median HCT $36 \%$ vs $40.2 \%$ ) of our cohort of patients, and with lower median body weight of $56 \mathrm{~kg}$. From our analysis of final PV exchanged during TPE, close to $8 \%$ of the procedures performed had PV exchanged below 1.0 times of patient's EPV, a minimal PV recommended to be exchanged for best exchange efficiency. This was mainly due to the variation of Hct level among this group of patients during the course of TPE. This reduced voluminous exchange procedures which could have negatively affected the efficiency of the TPE, resulting in further needs for exchanges that would have incurred additional costs, time and risks. However, there is an emerging concept that efficacy of TPE therapy is not only determined by plasma exchange volume per therapy but also by the total PV per treatment course. ${ }^{24}$ The average 4.5 treatment sessions among our patients receiving TPE with $92 \%$ of the procedures having exchanged PV more than 1.0 times patient's EPV may be sufficiently efficacious. However, analysis of treatment efficacy and clinical outcome was not included in this study.

Reliability of the TPE machine and quality of the centrifugation sets provided by the manufacturer with risk mitigation strategies were high priority for the safety and success of the procedure. Notably, our TPE unit operated over the past 3 years with single centrifugal system machine. Throughout the service period, we recorded no device malfunction events. However, our TPE team detected incidences related to centrifugation sets installation errors, resulting in delay in procedure initiation. In one such incident, visible blood leak contamination was detected by nursing staffs in the rotating separation chamber during exchange following an error in centrifugation set installation. The high centrifugation frequency resulted in a crack within the tubing set after incorrect installation, forcing the procedure to be abandoned. TPE was later resumed on completion of decontamination procedure. All earlier technical problems did not result in any adverse events to patients or staff.

The practice of TPE in our centre still leaves much room for further improvement. Over the last 3 years, we performed less than 20 courses of TPE treatment per year, equivalent to not more than $100 \mathrm{TPE}$ procedures. This however is highly dependent on availability of nursing staffs, healthcare funding, number of admissions as well as patient's ability to afford. Acquisition of a second TPE machine and monitoring equipment require additional budget allocation from the local authorities. As of current non-acute set-up, our TPE unit could only support patients with stable haemodynamic and requiring no ventilation. To include such patients in the future, there is a need to upgrade existing infrastructure in our neurology ward.

\section{CONCLUSIONS}

In summary, our local TPE database provided information from various perspectives on TPE in Malaysia over the past 3 years. Performing TPE in a non-acute set-up by trained neurology nurses proved safe, with low rates of complications comparable with other established centres. Although rare, occurrence of several technical errors and adverse effects that have not significantly improved over time require further quality improvement including staff training, treatment protocol revision and establishment of a more robust TPE registry. Important aspects of improvement should focus on enhancement of theoretical knowledge and practical experience, recognising complications and initiation of appropriate measures. 
Contributors All authors were involved in the patients' care. KSF and PYW identified the study subjects and collected the study data. FLH designed and conceptualised study, analysed the data, drafted manuscript for intellectual content. $\mathrm{FLH}$ is the guarantor. All authors made critical revisions and approved the final version to be published.

Funding The authors have not declared a specific grant for this research from any funding agency in the public, commercial or not-for-profit sectors.

Competing interests None declared.

Patient consent for publication Not required.

Ethics approval This study was approved by the Medical Research and Ethics Committee, Ministry of Health Malaysia and registered under the National Medical Research Registry (NMRR-11-1049-10503).

Provenance and peer review Not commissioned; externally peer reviewed.

Data availability statement Data are available upon reasonable request. All data relevant to the study are included in the article or uploaded as supplementary information. Data are available in the department of neurology, Hospital Kuala Lumpur Malaysia. Data contain deidentified data and are available on request from FLH at hiewf|@gmail.com.

Open access This is an open access article distributed in accordance with the Creative Commons Attribution Non Commercial (CC BY-NC 4.0) license, which permits others to distribute, remix, adapt, build upon this work non-commercially, and license their derivative works on different terms, provided the original work is properly cited, appropriate credit is given, any changes made indicated, and the use is non-commercial. See: http://creativecommons.org/licenses/by-nc/4.0/.

ORCID iD

Fu Liong Hiew http://orcid.org/0000-0002-4708-3734

\section{REFERENCES}

1 Reeves HM, Winters JL. The mechanisms of action of plasma exchange. Br J Haematol 2014;164:342-51.

2 Kaplan AA. Therapeutic plasma exchange: a technical and operational review. J Clin Apher 2013;28:3-10.

3 Winters JL. Plasma exchange: concepts, mechanisms, and an overview of the American Society for apheresis guidelines. Hematology Am Soc Hematol Educ Program 2012;2012:7-12.

4 Padmanabhan A, Connelly-Smith L, Aqui N, et al. Guidelines on the Use of Therapeutic Apheresis in Clinical Practice - Evidence-Based Approach from the Writing Committee of the American Society for Apheresis: The Eighth Special Issue. J Clin Apher 2019;34:171-354.

5 Wingerchuk DM, Weinshenker BG. Neuromyelitis optica (Devic's syndrome). Handb Clin Neurol 2014;122:581-99.

6 Willison $\mathrm{HJ}$, Jacobs BC, van Doorn PA. Guillain-Barré syndrome. Lancet 2016;388:717-27.
7 Hehir MK, Silvestri NJ. Generalized myasthenia gravis: classification, clinical presentation, natural history, and epidemiology. Neurol Clin 2018;36:253-60.

8 Lemaire A, Parquet N, Galicier L, et al. Plasma exchange in the intensive care unit: technical aspects and complications. J Clin Apher 2017;32:405-12.

9 Viswanathan S, Hiew FL. The establishment of in-house neurology driven therapeutic plasma exchange infrastructure in a resourcelimited public hospital in Malaysia: adopting and integrating evidenced-based health care technology through time. J Clin Apher 2019;34:434-44.

10 Mokrzycki MH, Balogun RA. Therapeutic apheresis: a review of complications and recommendations for prevention and management. J Clin Apher 2011;26:243-8.

11 Hakim RM, Siami GA. Plasmapheresis. In: Daugirdas JT, Ing TS, eds. Handbook of dialysis. 2nd edn. Boston: Little, Brown and Company, 1994: 220.

12 Norda R, Stegmayr BG, Group SA. Therapeutic apheresis in Sweden: update of epidemiology and adverse events. Transfus Apher Sci 2003;29:159-66.

13 Tombak A, Uçar MA, Akdeniz A, et al. Therapeutic plasma exchange in patients with neurologic disorders: review of 63 cases. Indian J Hematol Blood Transfus 2017;33:97-105.

14 Palma-Garcia L, Velásquez-Rimachi V, Pezo-Pezo A, et al. Therapeutic plasma exchange: experience in a third level Hospital, 2013-2016, lima (Peru). J Clin Apher 2018;33:480-5.

15 Narciso CT. Apheresis in the Philippines. J Clin Apher 2005;20:107-12.

16 Hiew FL, Ramlan R, Viswanathan S, et al. Guillain-Barré Syndrome, variants \& forms fruste: Reclassification with new criteria. Clin Neurol Neurosurg 2017;158:114-8.

17 Hung SKY, Hiew FL, Viswanathan S, et al. Conventional and unconventional therapies in typical and atypical chronic inflammatory demyelinating polyneuropathy with different clinical course of progression. J Peripher Nerv Syst 2018;23:183-9.

18 Kawachi I. Clinical characteristics of autoimmune optic neuritis. Clinical and Experimental Neuroimmunology 2017;8:8-16.

19 Stegmayr B, Ptak J, Wikström B, et al. World apheresis registry 2003-2007 data. Transfus Apher Sci 2008;39:247-54.

20 Main S. Expanding nursing knowledge for therapeutic plasma exchange a literature review paper. Ransfus Apher Sci 2017;56:774-7.

21 Schmidt JJ, Asper F, Einecke G, et al. Therapeutic plasma exchange in a tertiary care center: 185 patients undergoing 912 treatments - a one-year retrospective analysis. BMC Nephrol 2018;19:12.

22 Kes P, Janssens ME, Bašić-Jukić N, et al. A randomized crossover study comparing membrane and centrifugal therapeutic plasma exchange procedures. Transfusion 2016;56:3065-72.

23 Hafer C, Golla P, Gericke M, et al. Membrane versus centrifugebased therapeutic plasma exchange: a randomized prospective crossover study. Int Urol Nephrol 2016;48:133-8.

24 Hafer C, Kielstein JT. Pro: high dose of therapeutic plasma exchange-mind the gap! Nephrol Dial Transplant 2017;32:1457-60. 